\title{
Preface to special issue: Developments In Computational Models 2010
}

\author{
S. BARRY COOPER ${ }^{\dagger}$, ELHAM KASHEFI \\ and PRAKASH PANANGADEN ${ }^{\S}$ \\ ${ }^{\dagger}$ University of Leeds, Leeds, United Kingdom \\ Email: barry@amsta.leeds.ac.uk \\ $¥$ University of Edinburgh, Edinburgh, United Kingdom \\ Email: ekashefi@inf.ed.ac.uk \\ $\S_{\text {McGill University, Montreal, Canada }}$ \\ Email: prakash@cs.mcgill.ca \\ Received 16 January 2012
}

The scope of computation has expanded dramatically beyond the rubric of discrete, deterministic sequential computation under which it has been studied for many decades. That focus, of course, led to a great deal of deep and beautiful theory, but our focus in this special issue of Mathematical Structures in Computer Science is on new directions that have emerged from the study of computational phenomena in other settings, and thus on a celebration of the diversity of ideas, methods, new applications and novel sources of inspiration that have marked the modern era. The papers in this issue come from sources extending far beyond the core of computer science, yet using many of the central ideas that have evolved within computer science and mathematics. The nexus of all this activity has been, on the one hand, the boundary between logic and computation, and, on the other hand, the natural sciences, particularly physics and biology. The papers in this collection are expanded versions of selected papers from the DCM 2010 workshop, which was held in Edinburgh in July 2010. The theme of the workshop was Causality, Computation and Physics.

The dramatic developments in the algorithmic aspects of quantum computation that occurred in the mid 1990s are well known. More recently, ideas from the structural side of theoretical computer science have begun making an impact. The impetus for this work has come from the development of categorical quantum mechanics by Abramsky, Coecke, Selinger and others, and from the emergence of the so-called one-way model of quantum computation due to Rausendorff and Breigel, which has inspired a number of researchers to apply ideas from algebra, semantics and termrewriting theory. The collection of papers in this special issue shows the fertility of these ideas.

The papers by Murao and Soeda, Markham et al. and Dunjko and Kashefi included in this issue are, in one way or another, connected with the one-way model. There is also a paper on quantum computation by Floess et al. that is based on more traditional 
computer science topics, in this case boolean functions, but which also pushes back the boundaries by considering how learning and testing are affected by the ability to use quantum algorithms. The categorical perspective is well represented by the paper by Dixon and Kissinger, which combines categorical semantics, or, more precisely, the diagrammatic calculi that have evolved from it, with techniques for automated reasoning. The connection with the semantics of programming languages is represented by a paper by Valiron on a computational $\lambda$-calculus that was in part inspired by his work with Selinger on higherorder calculi for quantum programming. All these papers are excellent exemplars of the results that come from interactions across conventional academic boundaries: indeed, four of the papers are written by physicists employed as such.

We are particularly pleased to include the long contribution by Hardy. This paper addresses the core issues of the theme of the workshop, and is part of Hardy's ambitious programme to reconstruct quantum mechanics from a few basic principles.

Another and equally exciting strand of work represented in this issue is the connection with systems biology. We use this term to distinguish it from the use of standard algorithmic techniques to address problems in biology like gene sequencing. What the papers included here represent, by contrast, is the use of modelling techniques coming from theoretical computer science for modelling biological systems. There are four papers in this group, ranging from the explicitly biological to ones that abstract from the biological context and develop modelling tools. Cardelli's paper, which is based on his invited talk, gives an account of how the basic actions possible in two-strand DNA molecules can define a calculus rich enough to model molecular programming. This is pure biology done with the tools of computer science. The paper by Danos et al. develops a precise semantics for the core of the Kappa modelling language. This formalism takes its inspiration from mainstream computer science topics, such as $\lambda$-calculus and term rewriting, and extends them with some new ideas that are needed for modelling biological systems. The paper by Oury and Plotkin develops a formalism based on term-rewriting, but adapted to stochastic multiset rewriting to capture features essential for biological modelling. A particularly intriguing paper by Danos and Oury takes ideas and inspiration from statistical physics and applies them to the behaviour of Petri nets with a view to applying the same ideas to biological modelling.

Finally, we have included a paper by Bartha that exposes the monoidal structure of Turing machines. While this paper does not mention quantum mechanics as such, there is a clear connection with the monoidal structure made explicit in the categorical formulation of quantum mechanics.

The editors would like to thank the DCM steering committee and programme committee: at least 25 people were involved in organising the workshop and selecting the papers from the submissions. The DCM meeting was part of the Federated Logic Conference (FLoC), which is held every four years; the web page for the DCM workshop is www1.maths.leeds.ac.uk/ pmt6sbc/DCM10/. We are also grateful to the FLoC organising committee for administrative and other support. Quantum Information Science Scotland (QUISCO) organised the second day of DCM 2010, with support from the Carnegie Trust, and it is a pleasure to thank them for their support. We 
would also like to thank Giuseppe Longo for the support and encouragement he has given in the production of this special issue. Finally, we are delighted to thank the workshop speakers and attendees who provided such a stimulating atmosphere for the meeting.

Guest editors of the special issue.

\section{S. Barry Cooper}

Elham Kashefi

Prakash Panangaden 